\title{
Bridging the gap between supernovae and their remnants through multi-dimensional hydrodynamic modeling
}

\author{
S. Orlando ${ }^{1}$, M. Miceli ${ }^{2,1}$ and O. Petruk ${ }^{1,3}$ \\ ${ }^{1}$ INAF-Osservatorio Astronomico di Palermo, Piazza del Parlamento 1, 90134 Palermo, Italy \\ ${ }^{2}$ Dip. di Fisica e Chimica, Univ. di Palermo, Piazza del Parlamento 1, 90134 Palermo, Italy \\ ${ }^{3}$ Inst. Appl. Probl. in Mechanics and Mathematics, Naukova Street, 3-b Lviv 79060, Ukraine
}

\begin{abstract}
Supernova remnants (SNRs) are diffuse extended sources characterized by a complex morphology and a non-uniform distribution of ejecta. Such a morphology reflects pristine structures and features of the progenitor supernova (SN) and the early interaction of the SN blast wave with the inhomogeneous circumstellar medium (CSM). Deciphering the observations of SNRs might open the possibility to investigate the physical properties of both the interacting ejecta and the shocked CSM. This requires accurate numerical models which describe the evolution from the SN explosion to the remnant development and which connect the emission properties of the remnants to the progenitor SNe. Here we show how multi-dimensional SN-SNR hydrodynamic models have been very effective in deciphering observations of SNR Cassiopeia A and SN 1987A, thus unveiling the structure of ejecta in the immediate aftermath of the SN explosion and constraining the 3D pre-supernova structure and geometry of the environment surrounding the progenitor SN.
\end{abstract}

Keywords. hydrodynamics - instabilities - shock waves - ISM: supernova remnants

\section{Introduction}

Supernova remnants (SNRs) are probably among the most beautiful objects which is possible to observe in the sky. Their beauty is mostly due to the complex morphology and highly non-uniform distribution of ejecta which characterize most of them. In fact the propagation of ejecta drives shocks back and forth in the interstellar medium (ISM) and through ejecta themselves, leading to features and complex structures virtually at all spatial scales. The physics governing SNRs is studied thanks to observations at all wavelength bands, from radio, to optical, to X- and $\gamma$-rays. Multi-wavelength observations allow us to study both the thermal and non-thermal emission arising from SNRs. The physical and chemical properties of the emitting plasma are inferred through the analysis of both low and high resolution spectra. The observations allow us to investigate the morphology and the projected distributions of properties in the remnants and to study the dynamics, energetics, and evolution of SNRs. Besides the possibility to investigate the physical processes at work in SNRs, there is a large consensus that the observations encode information also on the progenitor star and the SN explosion from which the remnant originates. Obvious questions are: What information might we hope to obtain by deciphering the observations? How can we decipher the observations?

In fact, the morphological properties of SNRs may reflect pristine structures and features of the progenitor SN explosion and the physical and chemical properties of the 
progenitor SN. Thus, investigating the intimate link that exists between the morphological properties of a SNR and the complex phases in the SN explosion may allow us to trace back the structure and chemical composition of SN ejecta, and the dynamics and energetics of the SN explosion. An example is the SNR Cassiopeia A (Cas A) whose complex morphology is mainly due to a highly inhomogeneous distribution of ejecta determined most likely in the immediate aftermath of the SN explosion. In the case of young remnants, the morphological properties of SNRs may also reflect the early interaction of the SN blast with the inhomogeneous circumstellar medium (CSM) formed during the latest stages of the stellar progenitors evolution. In this case, analyzing the morphology of a SNR may help us to probe the structure and geometry of the CSM immediately surrounding the SN, providing important clues on the final stages of stellar evolution. An example is the remnant of SN 1987A whose morphology reflects the interaction of the blast wave with the inhomogeneous CSM consisting mainly of an H II region and a dense equatorial ring.

All the above considerations point out that linking SNR morphology to their SN progenitors is a necessary step: 1) to probe the physics of SN engines by providing insight into the asymmetries that occur during the SN explosion, and 2) to investigate the final stages of stellar evolution by unveiling the structure of the medium immediately surrounding the progenitor star. This step is now essential to open new exploring windows on SN and SNR issues especially in view of the spatially resolved high-resolution spectroscopy capability of the forthcoming Athena satellite (Nandra et al. 2013). In the following, we review our recent achievements in this field.

\section{The strategy to link supernovae to supernova remnants}

How to link SNe to SNRs? In general existing hydrodynamic and magnetohydrodynamic models have described either the SN evolution or the expansion of the remnant. Former models describe the complex phases of the SN evolution and they do not follow its subsequent transition to the phase of SNR and the interaction between the SN blast wave and the CSM. Conversely, models of SNRs describe the interaction of the remnant with the environment; however they usually assume an initial parameterized ejecta profile, typically few years after the SN event, and leave out an accurate description of the ejecta structure and chemical stratification soon after the SN explosion. Unfortunately this sharp distinction between these two classes of models and the poor communication between the community studying the physics of SNe and that of SNRs have prevented so far to disentangle the effects of the initial conditions (namely the SN event) from those of the boundary conditions (namely the interaction of the SN blast wave with the environment).

In fact there are several criticalities in describing the whole phenomenon from the onset of the supernova to the full remnant development. One serious problem is to catch the very different scales in time and space of SNe and SNRs. This has hampered so far to study the SN-SNR connection in detail. The core collapse of a massive star occurs on time scales of the order of a second and on dimensions of the order of $10000 \mathrm{~km}$ (e.g. Janka et al. 2016). At the other end, a young SNR evolves on time scales of the order of hundreds of years (if not thousands of years in the case of more evolved remnants) and can be characterized by dimensions of the order of parsecs. In addition the phenomenon is inherently three-dimensional (3D), thus requiring for the modeling a huge amount of numerical resources. This is the reason why the rare attempts to study the SN-SNR connection have been made, in the past, by adopting a one-dimensional (1D) approach (e.g. Badenes et al. 2008, Yamaguchi et al. 2014, Patnaude et al. 2015). However, the 3D 
nature of SN explosions is well known, being these characterized by strong asymmetries (jets, knots, clumps; e.g. Li et al. 1993, Wang et al. 2002, Kifonidis et al. 2006, Wang \& Wheeler 2008, Janka et al. 2016); hydrodynamic instabilities easily develop after the corecollapse, producing dense fingers of ejecta (e.g. Janka et al. 2016); and turbulence mixing of ejecta is most likely present (e.g. Janka et al. 2016). During the remnant expansion, the clumpy structure of the ejecta may induce complex interactions among the clumps and shrapnels of ejecta, enhancing the development of hydrodynamic instabilities, the mixing of initially chemically homogeneous layers, and some overturning of the ejecta due to hydrodynamic instabilities developing during clumps interaction (e.g. Orlando et al. 2012, Miceli et al. 2013). In addition the ambient medium (CSM or ISM) through which the SN blast wave expands is, in general, highly inhomogeneous and characterized, for instance, by nebulae, rings, clouds, or gradients of density. The ambient magnetic field is, in general, non-uniform; even in the case of a uniform magnetic field the obliquity angle between the magnetic field and the shock normal varies along the remnant outline (e.g. Petruk et al. 2009). Hydrodynamic instabilities develop at the contact discontinuity between the stellar debris and the shocked ambient medium and at the border of shocked CSM/ISM inhomogeneities (e.g. Orlando et al. 2005, Orlando et al. 2008).

In the attempt to study the SN-SNR connection, recently we have devised a strategy which is based on the coupling between a 1D model of core-collapse SN and a 3D model of SNR (Orlando et al. 2015, Orlando et al. 2016). The SN model describes the evolution of a core-collapse SN from the breakout of the shock wave at the stellar surface to the so-called nebular phase. The model is implemented through a relativistic, radiation-hydrodynamics Lagrangian code (Pumo \& Zampieri 2011) which includes a full general relativistic treatment and the radiative transfer coupled to relativistic hydrodynamics at all regimes. The model includes also the gravitational effects of the central compact remnant on the evolution of the ejecta (fall-back of material onto the compact remnant; amount of ejected ${ }^{56} \mathrm{Ni}$ ). The $1 \mathrm{D}$ SN model provides the initial radial distribution of ejecta for the 3D SNR model. The latter describes the transition from the phase of SN to that of SNR and the interaction of the remnant with the (inhomogeneous) ambient medium. The 3D SNR model is implemented with the FLASH code (Fryxell et al. 2000) and includes the effects of radiative losses from optically thin plasma, the deviations from equilibrium of ionization, the back reaction of accelerated cosmic rays, and the deviations from electron-proton temperature equilibration. The evolution of an appropriate post-explosion isotopic composition of the ejecta is accounted by performing multi-species simulations. The model describes the post-explosion structure of the ejecta by small-scale clumping of material and larger-scale anisotropies (see Fig. 1). The evolution of different plasma components (e.g. ejecta, inhomogeneities of CSM, etc.) is traced by considering passive tracers associated with each of them. From the model results, the thermal emission is synthesized in different bands. In this way, the model results can be compared with the observations to obtain a feedback on the SN-SNR models.

A major challenge in our approach is capturing the enormous range in time and space scales. The initial condition for the 3D simulations of the SNR is typically 1 day after the SN event. At that time the size of the ejecta distribution is of the order of tens of AU. Then the remnant evolution is followed for hundreds of years. At the final time, the size of the remnant can be of several pc. To catch this huge range of time and space scales, our approach exploits the adaptive mesh refinement capabilities of the FLASH code. About 20 nested levels of adaptive mesh refinement are typically used to reach an effective resolution of about $0.2 \mathrm{AU}\left(\approx 3 \times 10^{12} \mathrm{~cm}\right)$. This guarantees to have at least 100 computational cells per remnant radius during the whole evolution. 

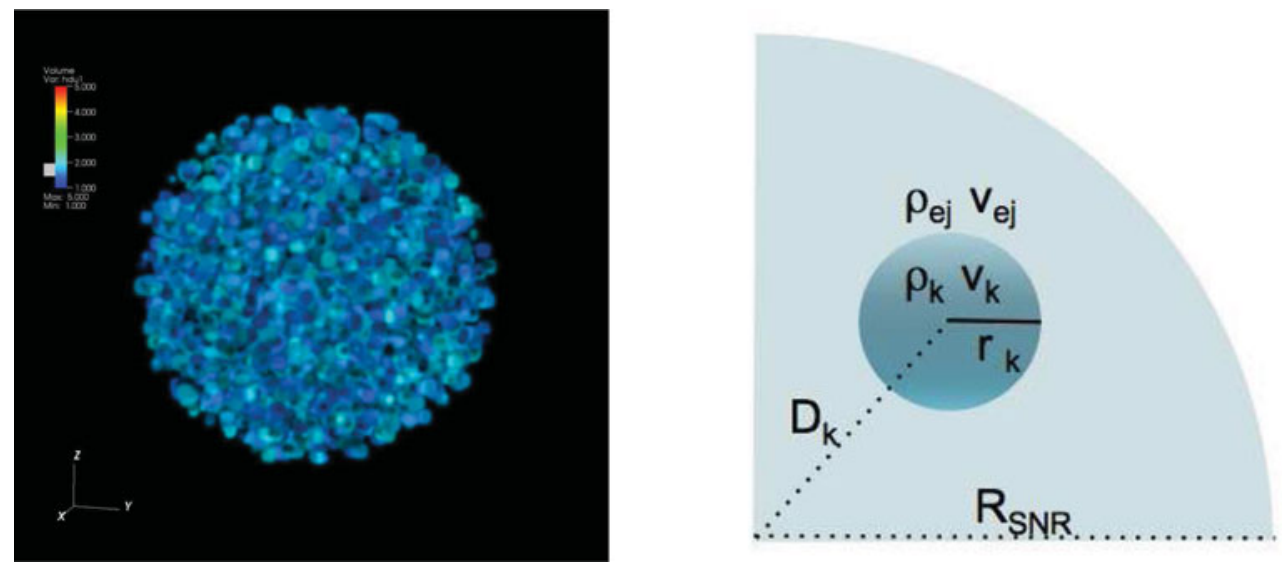

Figure 1. Initial 3D post-explosion structure of the ejecta. The panel on the left shows the small-scale clumping prescribed as in Orlando et al. (2012). The panel on the right shows a schematic view of the large-scale anisotropies described as overdense spherical knots characterized by radius, $r_{k}$, distance from the center of explosion, $D_{k}$, and density and velocity contrasts with respect to the surrounding ejecta, $\rho_{k} / \rho_{e j}$ and $v_{k} / v_{e j}$ respectively.

As first applications of our SN-SNR model, we have considered two study cases which are, in some way, complementary each other. The first is the SNR Cas A whose morphology is believed to reflect pristine structures and features developed in the immediate aftermath of the SN explosion. The second case is that of SN 1987A which is characterized by a very complex morphology which reflects the interaction of the SN blast wave with the strongly inhomogeneous CSM. These simulations required about 7.7 millions of CPU hours on the MareNostrum III cluster hosted at the Barcelona Supercomputing Center (Spain) and about 4 millions of CPU hours on the FERMI cluster hosted at CINECA (Italy).

\section{Effects of supernova anisotropies: The case of SNR Cassiopeia A}

The observations suggest that the morphology and expansion rate of Cas A are consistent with a remnant expanding through the wind of the progenitor red supergiant (Lee et al. 2014). Since the structure of the wind is expected to be spherically symmetric with the gas density proportional to $r^{-2}$ (where $r$ is the radial distance from the center of explosion), the complex morphology of the remnant and the highly non-uniform distribution of ejecta should originate from anisotropies developed soon after the SN explosion. For this reason, Cas A is considered a very attractive laboratory to bridge the gap between SNe and their remnants. In addition Cas A is one of the best studied SNR and its $3 \mathrm{D}$ structure has been characterized in good detail thanks to observations in the IR, optical, and X-ray bands (e.g. DeLaney et al. 2010, Hwang \& Laming 2012, Milisavljevic \& Fesen 2013). In particular, the observations show that the ejecta distribution is characterized by three extended Fe-rich regions and two Si-rich jets. In addition, rings of Si-, S-, and O-rich ejecta surround the Fe-rich regions (Milisavljevic \& Fesen 2013).

Recently we have investigated the origin of the most pronounced features observed in the actual distribution of ejecta in Cas A following the approach outlined in Sect. 2 (Orlando et al. 2016). First the 1D SN model was used to provide the initial radial profile of ejecta for the 3D SNR model, about 1 day after the SN event. Then the 3D post-explosion structure of the ejecta was described by small-scale clumping of material and larger-scale anisotropies (Kifonidis et al. 2006, Wang \& Wheeler 2008). The 

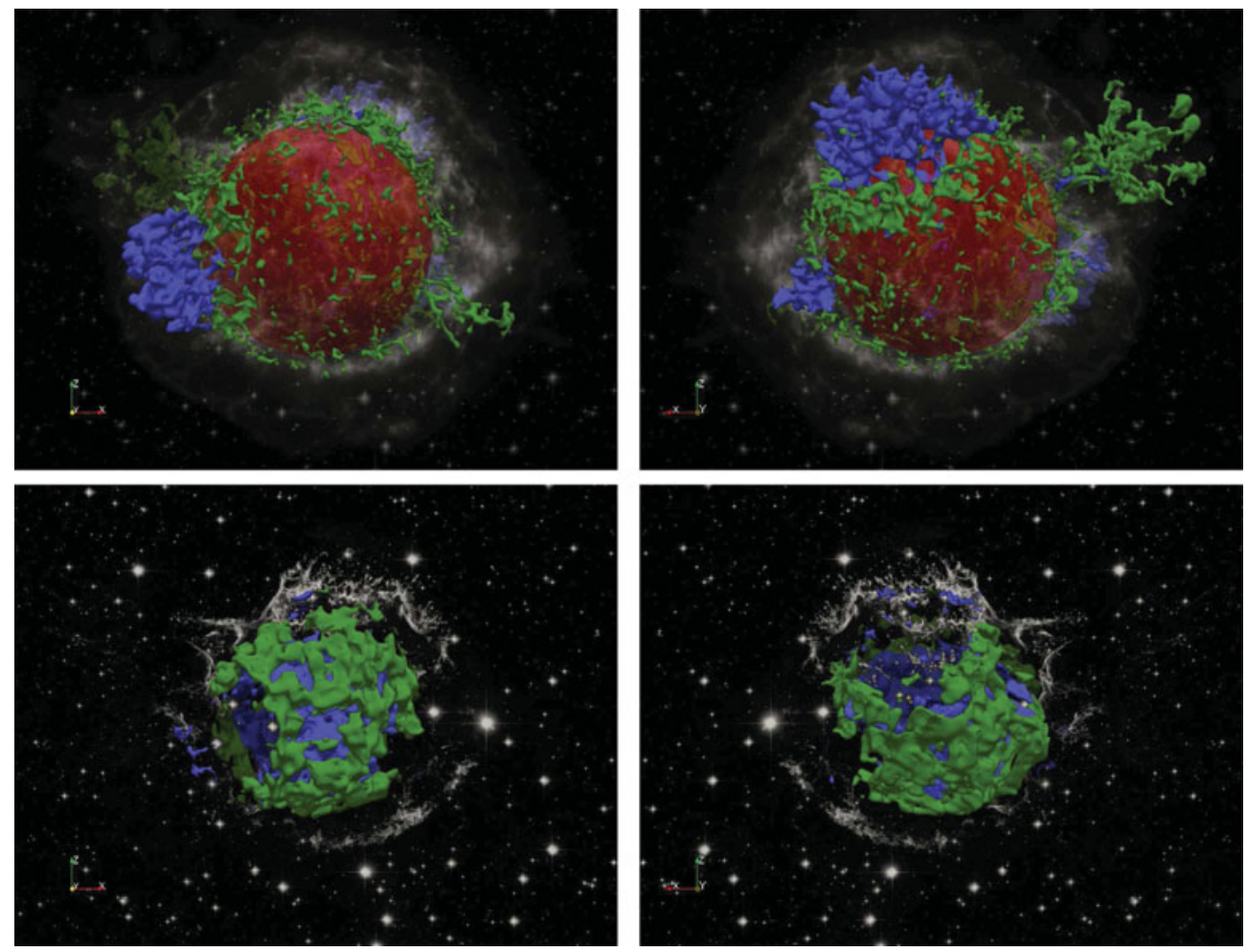

Figure 2. 3D spatial distribution of Cas A shocked (upper panels) and unshocked (lower panels) $\mathrm{Fe}$ (blue) and $\mathrm{Si} / \mathrm{S}$ (green) rich ejecta derived from the best-fit model (adapted from Orlando et al. 2016). The figure shows the 3D distributions assuming the perspective in the plane of the sky (on the left) or from behind Cas A (on the right). The transparent red sphere marks the fiducial reverse shock. The transparent image in the upper panels is a Chandra observation showing the hot shocked plasma in the wavelength band $[0.3,10] \mathrm{keV}$ (retrieved from www.nasa.gov); the transparent image in the lower panels is a composite Hubble Space Telescope (HST) image sensitive to emission in cold $\mathrm{O}$ and $\mathrm{S}$ lines (retrieved from www.spacetelescope.org).

small-scale clumping was prescribed as in Orlando et al. (2012, see left panel in Fig. 1). The large-scale anisotropies were described as overdense spherical knots characterized by the following parameters: radius of the knot, distance of the knot from the center of explosion, density and velocity contrasts of the knot with respect to the surrounding ejecta (see right panel in Fig. 1). By exploring the model parameters space, first we have derived the most likely mass of ejecta $\left(M_{\mathrm{ej}} \approx 4 M_{\odot}\right)$ and explosion energy $\left(E_{\mathrm{SN}} \approx 2.3 \times 10^{51} \mathrm{erg}\right)$ which are those able to reproduce simultaneously the radii and velocities of the forward and reverse shocks as observed at the current time (van Veelen et al. 2009) and the density of the shocked red supergiant wind inferred from observations (Lee et al. 2014). Then we have constrained the physical parameters (namely size, density, velocity, and position in the original onion-skin nucleosynthetic layering) characterizing the anisotropies developed soon after the SN explosion and responsible for the observed masses and distributions of $\mathrm{Fe}$ and $\mathrm{Si} / \mathrm{S}$ in Cas $\mathrm{A}$.

Figure 2 shows the distribution of both shocked and unshocked Fe-rich and Si/S-rich ejecta at the age of Cas A. We have found that post-explosion anisotropies (pistons) reproduce the observed distributions and masses of $\mathrm{Fe}$ and $\mathrm{Si} / \mathrm{S}$ if their total mass was $\approx 0.25 M_{\odot}$ (corresponding to $\approx 5 \%$ of the total mass of ejecta) and their kinetic energy $\approx 1.5 \times 10^{50} \mathrm{erg}$ (corresponding to $\approx 7 \%$ of the total energy of explosion). The model 
has shown that the pistons produce a spatial inversion of ejecta layers at the epoch of Cas A, leading to the Si/S-rich ejecta physically interior to the Fe-rich ejecta, in nice agreement with the observations of Cas A (DeLaney et al. 2010, Milisavljevic \& Fesen 2013). In addition the model explains the origin of the rings of Si/S-rich material encircling the Fe-rich regions (Milisavljevic \& Fesen 2013): they form at the intersection between the reverse shock and the material accumulated around the pistons during their propagation. As for the unshocked ejecta, the model has shown that the distribution of $\mathrm{Si} / \mathrm{S}$ is characterized by large cavities corresponding to the directions of propagation of the pistons/jets. This may explain why the cavities observed in near-IR observations are physically connected to the bright rings in the main-shell (Milisavljevic \& Fesen 2015). The model, therefore, supports the idea that the bulk of asymmetries observed in Cas A are intrinsic to the explosion.

It is interesting to note that, very recently, 3D simulations of a neutrino-driven SN explosion were able to reproduce some basic properties of Cas A (Wongwathanarat et al. 2016). The initial conditions of this model are given immediately after the core bounce and the simulations follow the evolution until $\approx 1$ day after the explosion, namely roughly the time when we have prescribed the post-explosion anisotropies in our 3D SNR simulations (Orlando et al. 2016). In one of their simulations, Wongwathanarat et al. (2016) have found three pronounced Fe-rich fingers that may correspond to the extended Fe-rich regions observed in Cas A. Interestingly their physical characteristics seem to be similar to those of initial pistons prescribed in our SN-SNR model, 1 day after the SN explosion.

\section{Effects of inhomogeneous CSM: The case of SN 1987A}

The effects of an inhomogeneous CSM on the morphology of a SNR can be investigated in SN 1987A. In fact, in this case, the surrounding medium (the nebula) is highly inhomogeneous as shown by optical images soon after the outburst. The nebula consists mainly of a dense central equatorial ring surrounded by an extended H II region. Two other rings lying in planes almost parallel above and below the equatorial one are also observed. This complex structure of the CSM is believed to originate from the interaction of a slow wind from the red supergiant phase with the faster wind from the blue supergiant phase (e.g. Morris \& Podsiadlowski 2007). According to most recent observations (Frank et al. 2016), currently the blast wave from the explosion has left the inner equatorial ring.

In a previous work, we have investigated the effects of the CSM on the morphology of the remnant of SN 1987A and the imprint of the SN on the X-ray emission of its remnant through hydrodynamic simulations (Orlando et al. 2015). The strategy is again that described in Sect. 2. The model describes the complex structure of the CSM accounting, in particular, for the HII region and the dense equatorial ring. We have explored the space of model parameters, searching for those best fitting the morphology, light curves, and spectra observed in the X-ray band. We have found that a model with explosion energy in the range $1.2-1.4 \times 10^{51} \mathrm{erg}$ and envelope mass in the range $15-17 M_{\odot}$ is able to reproduce the observed bolometric lightcurve of SN 1987A during the first 250 days after the SN event. The left panel of Fig. 3 shows the 3D volume rendering of the particle density of the shocked plasma at $t=30 \mathrm{yr}$ for the best-fit model in Orlando et al. (2015). This model predicts a total mass of the ring $M_{\mathrm{rg}}=0.062 M_{\odot}$ of which $\sim 64 \%$ is plasma with density $n \approx 1000 \mathrm{~cm}^{-3}$ and $\sim 36 \%$ is plasma with $n \approx 2.5 \times 10^{4} \mathrm{~cm}^{-3}$. Interestingly, these values are in very nice agreement with those derived for the density structure of the ring from the analysis of optical observations (Mattila et al. 2010). The model also predicts that the blast wave has recently left the dense ring and is now propagating in 

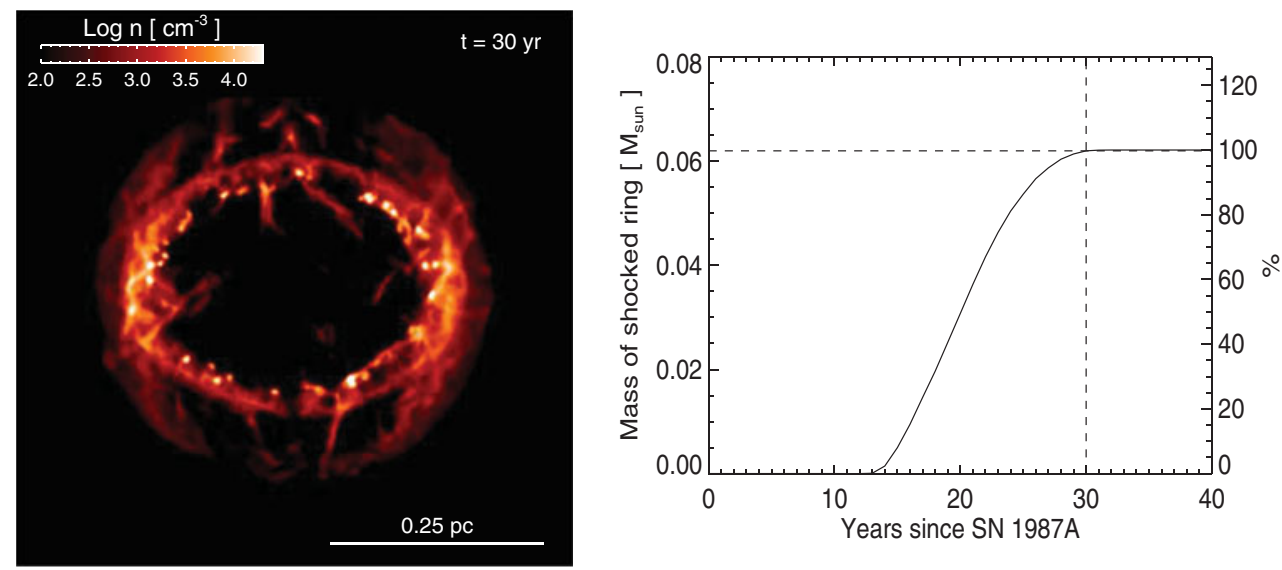

Figure 3. Left panel: three-dimensional volume rendering of the particle density of the shocked plasma during the interaction of the blast wave with the nebula, 30 years after the SN event (from the best-fit model of Orlando et al. 2015). Right panel: mass of the shocked ring vs. time; the vertical dashed line marks 30 years after the SN event; the horizontal dashed line indicates that the whole ring has been shocked.

a less dense environment (see right panel in Fig. 3). Again this is in excellent agreement with the recent findings from the analysis of X-ray observations (Frank et al. 2016) and, also, with the fading of the ring and implied destruction inferred from optical observations (Fransson et al. 2015).

From the model results, we have synthesized the X-ray emission, deriving light curves, images, and spectra to be compared with actual observations. Figure 4 shows the light curves derived from our best-fit model and the comparison with those observed. We have found that the same model fitting the bolometric lightcurve of the $\mathrm{SN}$ is also able to fit both the soft $([0.5,2.0] \mathrm{keV})$ and hard $([3.0,10] \mathrm{keV})$ X-ray light curves as derived from multi-epoch observations with ROSAT, ASCA, Chandra, and XMM-Newton Xray telescopes. In particular, the predictions of the model fit the most recent Chandra observations (Frank et al. 2016), supporting the idea that the blast wave has now left the dense ring. This study has demonstrated, for the first time, the consistency between a physical model reproducing the observables of the SN (namely the cause) and that reproducing the observables of the resulting remnant (namely the effect).

The model includes also some passive tracers to follow the evolution of the different plasma components (namely ejecta, H II region, and dense ring) and their contribution to X-ray emission (see Figs. 4 and 5). We have identified three main phases in the evolution of the remnant of SN 1987A. The first starts when the ejecta reach the H II region and last for about 13 years. In this phase the emission is dominated by the shocked H II region with a smaller contribution from shocked ejecta. Then the blast wave starts to propagate through the dense ring $(\approx 13$ yr after the SN event) and the X-ray emission becomes largely dominated by the shocked plasma from the ring (see Fig. 5). In this phase the contribution from shocked ejecta continue to increase faster than that of shocked plasma from the HII region, but still much lower than the contribution from shocked plasma from the ring (see Fig. 4). The model predicts that the blast wave has left the disk in the last few years and is now propagating through a less dense medium. As a consequence, the contribution from shocked ring will shortly decrease while the contribution from shocked ejecta will gradually increase becoming the dominant component in about $4-5$ years (see Fig. 4). During the first phase (H II region dominated), we have found that the 

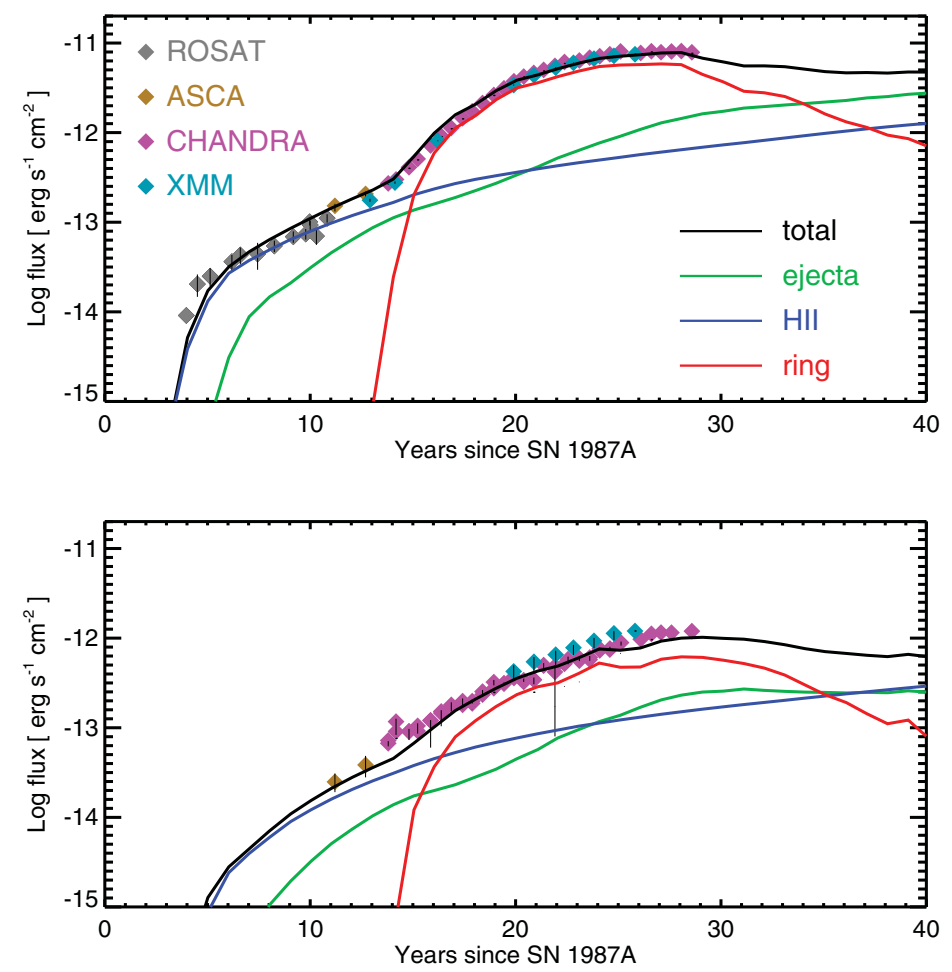

Figure 4. Observed and modeled X-ray light curves in the soft $([0.5,2.0] \mathrm{keV}$; upper panel) and hard $([3.0,10] \mathrm{keV}$; lower panel) bands (adapted from Orlando et al. 2015). Green, blue, and red lines mark the contribution to emission from the shocked ejecta, the shocked plasma from the H II region, and the shocked plasma from the ring, respectively.

shape of the X-ray lightcurve reflects the structure of the outer ejecta thus identifying the imprint of SN 1987A on the X-ray emission of its remnant. In the second phase (ring dominated), the comparison between model results and observations allowed us to constrain the structure and geometry of the CSM (see Fig. 5). In the third phase (ejecta dominated), expected in the next future, our model predicts that we will be able to investigate the structure and chemical stratification of the ejecta.

\section{Conclusions}

There is a growing interest in Astrophysics on studies aimed at linking the morphological properties of SNRs to their progenitor SNe. In fact SNRs morphology and properties are expected to reflect the physical and chemical properties of the progenitor SNe and of the environment in which blast waves travel. Multi-wavelength/multi-messenger observations of SNRs, therefore, should encode information about the physical and chemical properties of both the stellar debris and the surrounding CSM. In the first case, we could have the possibility to investigate the energetics and dynamics of the SN progenitor and the anisotropies developed during the complex phases of the SN explosion; in the latter we could derive important clues on the final stages of stellar evolution. Linking SNe to SNRs, therefore, has breakthrough potential to open new exploring windows on SN and SNR issues, especially in view of the spatially resolved high-resolution spectroscopy capability of the forthcoming Athena satellite (Nandra et al. 2013). 


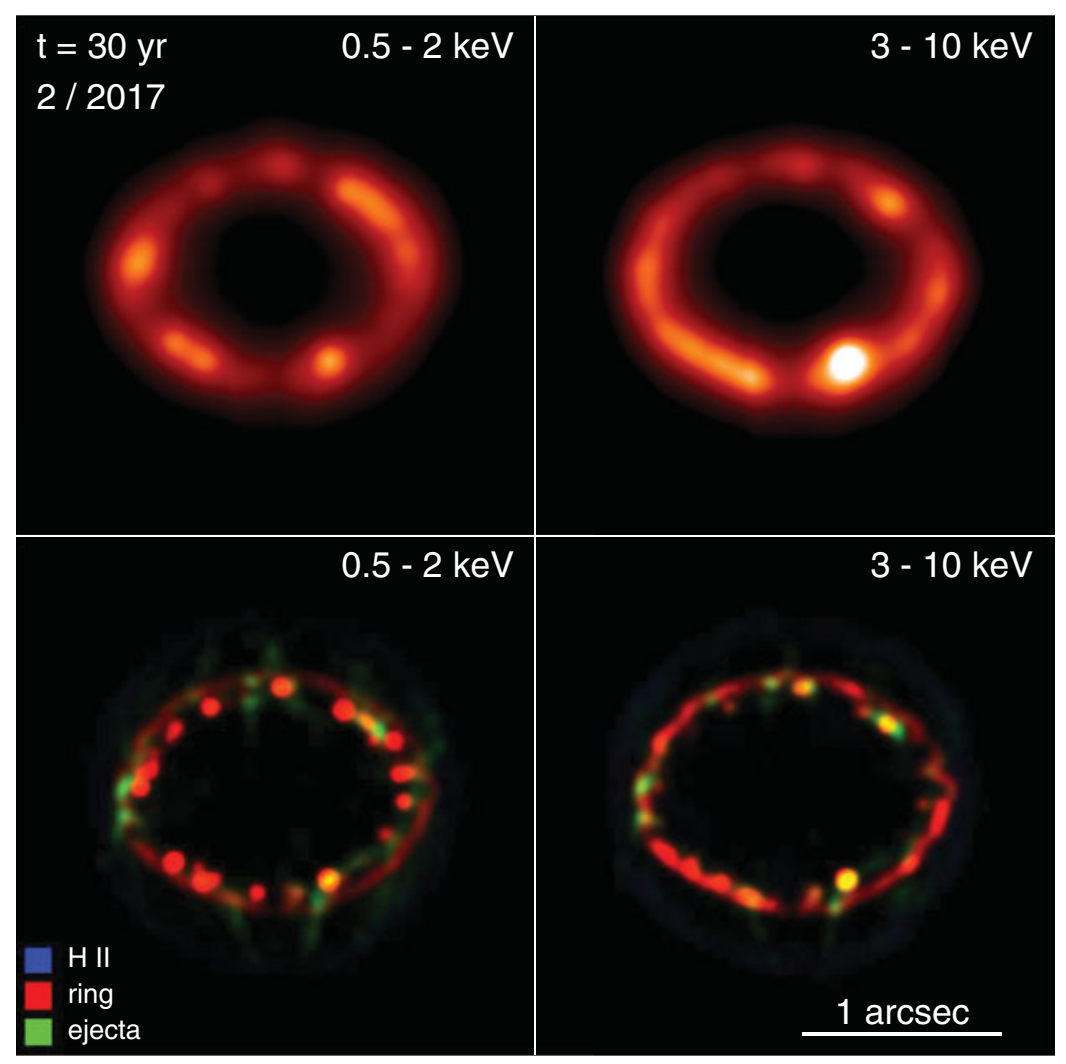

Figure 5. Synthetic maps of X-ray emission in the soft $([0.5,2.0] \mathrm{keV}$; on the left) and hard $([3.0,10] \mathrm{keV}$; on the right) bands integrated along the line of sight, 30 years after the SN event (from the best-fit model of Orlando et al. 2015); each image has been normalized to its maximum for visibility. In the upper panels the images have been convolved with a Gaussian of size 0.15 arcsec to approximate the spatial resolution of Chandra observations. In the lower panels, the figure shows three-color composite images of the X-ray emission smoothed with a Gaussian of size 0.025 arcsec; the colors in the composite show the contribution to emission from the different shocked plasma components, namely the ejecta (green), the ring (red), and the $\mathrm{H}$ II region (blue).

In this contribution we have reviewed our recent achievements in the study of the SNSNR connection. In particular, we have devised a strategy based on 3D hydrodynamic models to link the morphological properties observed in SNRs to the structure of the ejecta soon after the progenitor SN explosions and to the structure of the environment in which the remnant expands. In the case of Cas A, we have shown that our approach was very effective in deriving the physical properties of the main anisotropies developed in the aftermath of the SN explosion and responsible for the observed non-uniform distribution of ejecta. In the case of SN 1987A, we have demonstrated that the physical model reproducing the main observables of the $\mathrm{SN}$ reproduces also the X-ray emission of the subsequent expanding remnant, thus bridging the gap between SNe and SNRs. The study was very effective also to identify the imprint of SN 1987A on the X-ray emission of its remnant and to constrain the structure and geometry of the surrounding nebula, formed in the latest stage of the star progenitor's evolution.

Our study has shown that detailed and complete numerical models accounting for the 3D nature of the phenomenon are necessary to successfully decipher the observations and, 
therefore, to connect the observed morphology of SNRs to the physics of the progenitor SN engine. However, deciphering observations might depend critically on the models. The latter should connect the stellar progenitor, the SN, and the SNR self-consistently. The models should be guided by observational facts and they should account for the dynamics, energetics, and spectral properties of SNe and SNRs. Finally it is highly desirable to strongly improve the synergy and communication between the SN and SNR communities. The IAU Symposium 331, bringing together theorists and observers from these communities, is an excellent example of how to accomplish this task.

\section{Acknowledgements}

The authors acknowledge partial support from the IAU to participate to the IAU Symposium 331. This paper was partially funded by the PRIN INAF 2014 grant "Filling the gap between supernova explosions and their remnants through magnetohydrodynamic modeling and high performance computing". The software used in this work was, in part, developed by the U.S. Department of Energy-supported Advanced Simulation and Computing/Alliance Center for Astrophysical Thermonuclear Flashes at the University of Chicago. We acknowledge that the results of this research have been achieved using the PRACE Research Infrastructure resource MareNostrum III based in Spain at the Barcelona Supercomputing Center (PRACE Award N.2012060993) and the FERMI cluster based in Italy at CINECA (ISCRA Award N. HP10BI36DG,2012).

\section{References}

Badenes, C., Hughes, J. P., Cassam-Chenaï, G., \& Bravo, E. 2008, ApJ, 680, 1149

DeLaney, T. et al. 2010, ApJ, 725, 2038

Frank, K. A., Zhekov, S. A., Park, S., et al., 2016, ApJ, 829, 40

Fransson, C., Larsson, J., Migotto, K., et al., 2015, ApJ, 806, L19

Fryxell, B. et al. 2000, ApJS, 131, 273

Hwang, U. \& Laming, J. M. 2012, ApJ, 746, 130

Janka, H.-T., Melson, T., \& Summa, A. 2016, Ann. Rev. Nucl. Part. Sci., 66, 341

Kifonidis, K., Plewa, T., Scheck, L., Janka, H.-T., \& Müller, E. 2006, A\&̈A, 453, 661

Lee, J.-J., Park, S., Hughes, J. P., \& Slane, P. O. 2014, ApJ, 789, 7

Li, H., McCray, R., \& Sunyaev, R. A. 1993, ApJ, 419, 824

Mattila, S., Lundqvist, P., Gröningsson, P., et al., 2010, ApJ, 717, 1140

Miceli, M., Orlando, S., Reale, F., Bocchino, F., \& Peres, G. 2013, MNRAS, 430, 2864

Milisavljevic, D. \& Fesen, R. A. 2013, ApJ, 772, 134

Milisavljevic, D. \& Fesen, R. A. 2015, Science, 347, 526

Morris, T. \& Podsiadlowski, P. 2007, Science, 315, 1103

Nandra, K. et al. 2013, ArXiv e-prints: 1306.2307

Orlando, S., Peres, G., Reale, F., et al., 2005, A\&A, 444, 505

Orlando, S., Bocchino, F., Reale, F., Peres, G., \& Pagano, P., 2008, ApJ, 678, 274

Orlando, S., Bocchino, F., Miceli, M., Petruk, O., \& Pumo, M. L. 2012, ApJ, 749, 156

Orlando, S., Miceli, M., Pumo, M. L., \& Bocchino, F. 2015, ApJ, 810, 168

Orlando, S., Miceli, M., Pumo, M. L., \& Bocchino, F. 2016, ApJ, 822, 22

Patnaude, D. J., Lee, S.-H., Slane, P. O., et al., 2015, ApJ, 803, 101

Petruk, O., Dubner, G., Castelletti, G., et al., 2009, MNRAS, 393, 1034

Pumo, M. L. \& Zampieri, L. 2011, Astrophys. J., 741, 41

van Veelen, B., Langer, N., Vink, J., et al., 2009, A\&SA, 503, 495

Wang, L. et al. 2002, ApJ, 579, 671

Wang, L. \& Wheeler, J. C. 2008, ARA\&A, 46, 433

Wongwathanarat, A., Janka, H.-T., Mueller, E., et al., 2016, ArXiv e-prints, 1610.05643

Yamaguchi, H. et al. 2014, ApJ, 785, L27 